\title{
ESTUDOS DA AFLATOXINA NO AMENDOIM, DA COLHEITA À INDUSTRIALIZAÇÃ̃O, NA REGIÃO DE MONTE ALTO, S. P.*
}

HoMero FoNSECA **

\section{RESUMO}

Neste trabalho foi investigada, na região de Monte Alto, S.P., a incidência de aflatoxina no amendoim (Arachis hypogaea L.) em três Estágios de seu ciclo de industrialização: a) ao ser entregue à fábrica, Epocas I e II; b) durante seu armazenamento, Epocas III e IV e c) após a extração do óleo (farelo), Epocas V e VI. Em cada Estágio foram feitas duas coletas de 10 amostras cada, num total de 40 amostras de amendoim e 20 de farelo.

Dos resultados pôde-se concluir que: 1) a maioria das amostras, representando $90 \%$ do total, continha aflatoxina; 2) o nível, em termos de aflatoxina $\mathrm{B}_{1}$, foi elevado, com $60 \%$ das amostras ultrapassando 1,00 ppm., na categoria "Muito Alta"; 3) os níveis subiram da Epoca I até a Epoca IV; médias de 0,10 a 2,49 ppm, decrescendo nas Epocas V e VI: 1,02 e 1,25 ppm, com média geral de $1,23 \mathrm{ppm}$; 4) o lavrador entrega amendoim já tóxico à fábrica; 5) o armazenamento na fábrica parece contribuir para o aumento do nível de afaltoxina.

\section{INTRODUÇÃO}

A história da aflatoxina começou a ser contada quando STEVENS et alii (1960) descreveram o aparecimento de uma nova doença, em peruzinhos, nas granjas inglesas, doença essa que matava as aves dentro de uma semana.

Veterinários e pesquisadores ingleses batizaram-na de doença " $\mathrm{X}$ " dos perus e foi a responsabilizada pela morte de mais de 100.000 peruzinhos entre maio e agosto de 1960 (BLOUNT, 1961).

Verificou-se que as rações contendo torta de amendoim eram o fator comum em todos os surtos (ASPLIN e CARNAGHAN, 1961) tendo sido

\footnotetext{
* Entregue para publicação em 10/12/1976

* Departamento de Tecnologia Rural
} 
posteriormente constatado que o responsável pela mortandade eram alguns metabólicos altamente tóxicos produzidos pelo fungo Aspergillus flavus (SARGEANT et alii, 1961) que se desenvolvia sobre o amendoim após a colheita em condições favoráveis de umidade e temperatura. As quatro substâncias tóxicas isoladas inicialmente foram denominadas de aflatoxinas $B_{1}, B_{2}, G_{1}$ e $G_{2}$ (por causa das suas florescências azuis e esverdeadas) sabendo-se hoje da existência de outros metabólicos denominados $\mathbf{M}_{1}, \mathbf{M}_{2}, \mathbf{B}_{2 \mathrm{a}}, \mathrm{G}_{2 \mathrm{a}}, \mathrm{GM}_{1}$ e $\mathrm{B}_{3}$ (DUTTON e HEATHCOTE, 1969).

A incidência de aflatoxina em nosso Estado tem sido grande, conforme o demonstram os trabalhos de MENEZES et alii (1966), TANGO et alii (1967) e FONSECA (1968).

Em virtude da importância do problema resolvemos aprofundar nosso estudo procurando conhecer qual o estágio da agro-indústria do amendoim, dentro das condições prevalecentes em nosso Estado, em que havia maior incidência da aflatoxina: se com o lavrador - desde a colheita até o momento da entrega do amendoim na fábrica - ou se nas mãos da indústria - durante o armazenamento da matéria-prima e até o sub-produto estocado, que pode ser torta ou farelo. A região araraquarense foi escolhida pelo fato de, em pesquisa anterior (FONSECA, 1968), ter apresentado níveis gerais de aflatoxina mais elevados que em outras regiões, como também por ter sido mais elevada nesta região a incidência das aflatoxinas $\mathbf{G}$.

\section{MATERIAL E MÉTODO}

O material utilizado na execução deste trabalho constou de amostras de amendoim em casca e farelo de amendoim da safra das "águas" de 1967/68 coletadas na fábrica de óleo localizada no município de Monte Alto. A fábrica e as práticas agrícolas da região em que está situada têm as seguintes características predominantes: a fábrica tem capacidade de processamento de 90 toneladas de amendoim em casca por dia. O armazenamento da matéria-prima é feito a granel em grandes armazens os quais são bem protegidos contra a chuva. Todavia, quando há excesso de matéria-prima, esta é empilhada, ensacada ao ar livre e apenas coberta na parte superior com encerados (não foi o caso deste ano).

Nesta região o amendoim é colhido com o auxílio de sulcadores e deixado horizontalmente disposto, ou mesmo com as vagens para baixo, secando na leira por uns 2 ou 3 dias batendo-o, ensacando e entregando-a à fábrica imediatamente. Há pressa da retirada do amendoim do campo pois logo em seguida devem plantar o tomate que é a cultura principal.

As amostragens foram feitas nas seguintes datas: 


Época I:
Época II:
Época III:
Época IV:
Época V:
Época VI:

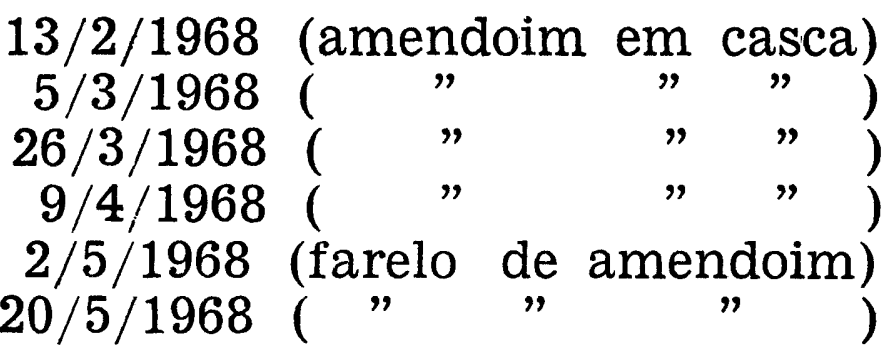

Em cada Época foram coletadas 10 amostras e numeradas, pela ordem de recolhimento, de 1 a 10, dando um total geral de 60 amostras. Nas Épocas I e II as 10 amostras foram retiradas de 10 caminhões diferentes à medida que iam chegando à fábrica. De cada um deles foi retirada uma amostra média, representando no mínimo 10 sacos. Estas duas Épocas compõem o Estágio 1.

Nas Epocas III e IV as amostras foram tomadas da matéria-prima estocada nos armazens tendo-se procurado retirar parcelas do maior número de sacos possível. Estas duas Épocas compõem o Estágio 2.

Nas Épocas V e VI foi usado o mesmo critério anterior, porém foram colhidas amostras de farelo de amendoim. Estas duas Épocas perfazem o Estágio 3.

Cada amostra de amendoim em casca foi constituída $5 \mathrm{~kg}$ e as de farelo, entre 2 e $3 \mathrm{~kg}$.

As amostras de amendoim foram descascadas e as amêndoas trituradas, em máquina de moer carne, bem homogeneizadas e peneiradas em peneiras de crivo de 1680 micra (10 "mesh") e a seguir desengorduradas em extrator de Soxhlet, por 8 horas. Os farelos foram também triturados em moinho de discos e peneirados.

A toxina foi extraída de acordo com o método de LEE (1965) e dosadas pelo método de COOMES e FEUELL (1965). As amostras foram analisadas em triplicata e os resultados expressam a média obtida nas três deetrminações.

Para enquadramento das amostras quanto à sua toxidez foi utilizada a tabela do TROPICAL PRODUCTS INSTITUTE (1962) e que está baseada nos efeitos de testes biológicos com marrequinhos de um dia (QUADRO I).

QUADRO I - Relação entre concentração de aflatoxina $B_{1}$ e toxidez.

\begin{tabular}{l|l}
\hline \multicolumn{1}{c|}{ Nível de aflatoxina $B_{1}$} & \multicolumn{1}{c}{ Categoria de toxidez } \\
\hline \hline Abaixo de $0,05 \mathrm{ppm}$ & Baixa ou negativa \\
Entre 0,05 e $0,25 \mathrm{ppm}$ & Média \\
Entre 0,25 e $1,00 \mathrm{ppm}$ & Alta \\
Acima de $1,00 \mathrm{ppm}$ & Muito alta \\
\hline
\end{tabular}


A umidade das amostras foi determinada em balanças do tipo ULTRA X com raios infra-vermelhos.

Para a análise da variância dos resultados, em termos de aflatoxina $\mathbf{B}_{1}$, foram tomados em cada amostra os valores encontrados para a aflatoxina $B_{1}$ somados à metade dos valores de $G_{1}$ visto que esta tem cerca de $50 \%$ da toxidez da $B_{1}$ (CARNAGHAN et alii, 1963). Estas variáveis $(\mathrm{y})$ foram transformadas em $\log _{10}(\mathrm{y}+1)$ de acordo com SNEDECOR (1956) e STEEL e TORRIE (1960) pois os valores encontrados eram de magnitudes muito diferentes e incluiam também valores iguais a zero.

Foi feito também um estudo da evolução do teor de aflatoxina nas amostras, em função do tempo, por meio de regressão. A soma dos Quadrados (SQ), para tempo, foi desdobrada num componente linear, num componente de $2 .^{\circ}$ grau e em desvios de regressão. Paralelamente foram estimadas as constantes de uma equação de regressão do tipo:

$\log _{10}(y+1)=a+b x+c^{2}$ (SNEDECOR, 1956)

onde $\mathrm{x}=\mathrm{n} .^{\circ}$ de dias a partir do início das coletas.

\section{RESULTADOS E DISCUSSÃO}

Os resultados obtidos nas análises das aflatoxinas $B_{1}$ e $G_{1}$ encontram-se nos QUADROS II e III, os da umidade no QUADRO IV e os das análises estatísticas nos QUADROS V, VI e VII.

QUADRO II - Teor das aflatoxinas $B_{1}$ e $G_{1}$ nas amostras de amendoim e farelo de amendoim nas diversas Épocas (expresso e mppm)

\begin{tabular}{|c|c|c|c|c|c|c|c|c|c|c|c|c|}
\hline \multirow{2}{*}{ Amostras } & \multicolumn{2}{|c|}{ Epoca I } & \multicolumn{2}{|c|}{ Epoca II } & \multicolumn{2}{|c|}{ Epoca III } & \multicolumn{2}{|c|}{ Epoca IV } & \multicolumn{2}{|c|}{ Epoca V } & \multicolumn{2}{|c|}{ Epoca VI } \\
\hline & $\mathrm{B}_{1}$ & $\mathrm{G}_{1}$ & $\mathrm{~B}_{1}$ & $\mathrm{G}_{1}$ & $\mathrm{~B}_{1}$ & $\mathrm{G}_{1}$ & $\mathrm{~B}_{1}$ & $\mathrm{G}_{1}$ & $\mathrm{~B}_{1}$ & $\mathrm{G}_{1}$ & $\mathrm{~B}_{1}$ & $\mathrm{G}_{1}$ \\
\hline 1 & 0,0 & 0,0 & 0,75 & 0,12 & 0,75 & 0,12 & 15,00 & 5,62 & 0,75 & 0,28 & 1,75 & 0,66 \\
\hline 2 & 0,02 & 0,01 & 7,50 & 2,81 & 7,50 & 2,81 & 0,75 & 0,28 & 1,75 & 0,12 & 1,75 & 0,66 \\
\hline 3 & 0,0 & 0,0 & 0,30 & 0,28 & 1,75 & 0,66 & 3,75 & 1,41 & 0,75 & 0,28 & 0,75 & 0,28 \\
\hline 4 & 0,08 & 0,12 & 0,0 & 0,12 & 0,75 & 0,28 & 1,75 & 0,0 & 0,75 & 0,28 & 0,75 & 0,28 \\
\hline 5 & 0,0 & 0,0 & 1,75 & 1,41 & 0,75 & 1,41 & 1,75 & 5,62 & 0,75 & 0,28 & 0,75 & 0,28 \\
\hline 6 & 0,0 & 0,01 & 0,30 & 0,01 & 0,30 & 0,12 & 1,75 & 2,81 & 1,75 & 0,12 & 0,75 & 0,28 \\
\hline 7 & 0,0 & 0,0 & 0,30 & 0,12 & 0,30 & 0,28 & 0,30 & 0,03 & 0,75 & 0,28 & 0,75 & 0,28 \\
\hline 8 & 0,75 & 0,28 & 1,75 & 1,41 & 15,00 & 1,41 & 1,75 & 0,28 & 0,75 & 0,28 & 0,75 & 0,28 \\
\hline 9 & 0,0 & 0,0 & 0,30 & 0,28 & 0,30 & 0,01 & 0,30 & 0,12 & 0,02 & 0,02 & 0,75 & 0,28 \\
\hline 10 & 0,0 & 0,0 & 1,75 & 0,28 & 0,75 & 0,66 & 0,30 & 0,12 & 0,75 & 0,12 & 0,75 & 0,28 \\
\hline
\end{tabular}


QUADRO III - Médias parciais e geral da aflatoxina $\left(B_{1}+1 / 2 G_{1}\right)$ por EPOCAS e por ESTÁGIOS, (expressas em ppm)

\begin{tabular}{|c|c|c|c|c|c|c|}
\hline \multicolumn{6}{|c|}{ EPOCA } & \multirow{2}{*}{$\begin{array}{l}\text { Média } \\
\text { Geral }\end{array}$} \\
\hline I & II & III & IV & V & VI & \\
\hline 0,10 & 1,38 & 1,99 & 2,49 & 1,02 & 1,25 & 123 \\
\hline \multicolumn{2}{|c|}{ ESTAGIO 1} & \multicolumn{2}{|c|}{ ESTÁGIO 2} & \multicolumn{2}{|c|}{ ESTAGIO 3} & \\
\hline \multicolumn{2}{|c|}{0,62} & \multicolumn{2}{|c|}{1,50} & \multicolumn{2}{|c|}{0,90} & \\
\hline
\end{tabular}

QUADRO IV - Teor de umidade das amostras (expresso em percentagens).

\begin{tabular}{|c|c|c|c|c|c|c|}
\hline \multirow{2}{*}{ Amostra } & \multicolumn{6}{|c|}{ EPOCA } \\
\hline & I & II & III & IV & V & VI \\
\hline 1 & 10,1 & $\overline{7,4}$ & 7,9 & 7,5 & 8,0 & $\overline{8,5}$ \\
\hline 2 & 7,5 & 10,4 & 10,0 & 8,1 & 7,5 & 9,1 \\
\hline 3 & 5,2 & 10,3 & 8,2 & 7,8 & 8,0 & 8,3 \\
\hline 4 & 12,5 & 10,1 & 7,9 & 8,6 & 8,4 & 7,9 \\
\hline 5 & 9,0 & 8,4 & 7,7 & 8,8 & 7,9 & 8,5 \\
\hline 6 & 11,2 & 8,2 & 8,4 & 7,4 & 8,0 & 9,0 \\
\hline 7 & 12,1 & 7,5 & 8,8 & 9,1 & 6,9 & 7,8 \\
\hline 8 & 7,6 & 9,8 & 9,2 & 7,9 & 7,7 & 8,7 \\
\hline 9 & 9,1 & 7,3 & 7,6 & 8,4 & 8,3 & 8,8 \\
\hline 10 & 6,5 & 6,8 & 8,2 & 7,8 & 7,7 & 7,8 \\
\hline
\end{tabular}

QUADRO V - Distrbiuição das amostras por níveis de aflatoxina $\left(B_{1}+1 / 2 G_{1}\right)$ e respectivas categorias de toxidez em números absolutos (n) e percentagens.

\begin{tabular}{|c|c|c|c|}
\hline Niveis (ppm) & $\mathrm{n}$ & $\%$ & Categoria de toxidez \\
\hline $0,0-0,05$ & 9 & 15,00 & Baixa ou negativa \\
\hline $0,05-0,25$ & 2 & 3,33 & Média \\
\hline $0,25-1,00$ & 13 & 21,67 & Alta \\
\hline $1,00-2,50$ & 27 & 45,00 & \\
\hline $2,50-5,00$ & 3 & 5,00 & Muito alta \\
\hline $5,00-10,00$ & 2 & 3,33 & \\
\hline Acima de 10,00 & 4 & 6,67 & \\
\hline TOTAL & 60 & 100,00 & \\
\hline
\end{tabular}


QUADRO VI - Valores de F obtidos na análise da variância

\begin{tabular}{l|c|c}
\hline \multicolumn{1}{c|}{ Tratamento } & Graus de Liberdade & $F$ \\
\hline \hline Epocas & $(5)$ & $3,29 * *$ \\
E. Estágios & 2 & $4,99 * *$ \\
D. Estágio 1 & 1 & $6,12 * *$ \\
D. Estágio 2 & 1 & 0,24 \\
D. Estágio 3 & 1 & 0,12 \\
\hline
\end{tabular}

** Significância ao nível de $1 \%$ de probabilidade.

QUADRO VII - Valores de F referentes à aplicação de regressão no estudo do efeito de Epocas.

\begin{tabular}{l|c|c}
\hline \multicolumn{1}{r}{ Tratamento } & Graus de Liberdade & $\mathrm{F}$ \\
\hline \hline Epocas & $(5)$ & $3,29 * *$ \\
Linear & 1 & 2,87 \\
Quadrático & 1 & $10,65 * *$ \\
Desvio Regressão & 3 & 0,98 \\
Resíduo & 54 & - \\
\hline
\end{tabular}

** Significância ao nível de $1 \%$ de probabilidade.

QUADRO I - Relação entre concentração de aflatoxina $B_{1}$ e toxidez

\begin{tabular}{cl|l}
\hline NíVEL DE AFLATOXINA & \multicolumn{1}{c}{ Categoria de toxidez } \\
\hline \hline Abaixo de $0,05 \mathrm{ppm}$ & Baixa ou Negativa \\
Entre 0,05 e $0,25 \mathrm{ppm}$ & Média \\
Entre 0,25 e $1,00 \mathrm{ppm}$ & Alta \\
Acima de $1,00 \mathrm{ppm}$ & Muito Alta \\
\hline
\end{tabular}

Pôde-se observar, pelos resultados, que a maioria das amostras $(90 \%)$ continha aflatoxina e em nível que pode-se considerar elevado pois à exceção da Época I todas as Épocas apresentaram níveis médios de aflatoxina acima de 1,00 ppm, considerado muito alto.

Pelo exame do QUADRO V pode-se observar que $60 \%$ das amostras apresentou mais de 1,00 ppm e portanto, enquadradas na categoria de toxidez "Muito Alta". Seis amostras (10\%) da Época I estavam completamente isentas de aflatoxina. 
O nível de aflatoxina cresceu da Época I até a Época IV (de 0,10 a 2,49 ppm) decrescendo nas Épocas V e VI (1,02 e 1,25 ppm). A média geral atingiu 1,23 ppm. Como pode-se observar as amostras da Época II já entraram na fábrica com um nível um pouco elevado de aflatoxina (média de 1,38 ppm) mas as amostras armazenadas à espera da industrialização apresentaram um nível bem mais elevado significando, a nosso ver, que o teor de umidade elevado, apresentado por muitas amostras (representando uma boa quantidade de material), nas Épocas I e II (QUADRO IV), dando condições de crescimento ao A. flavus, deve ter contribuído para o aumento do teor de aflatoxina nas amostras das Épocas III e IV. A indústria por sua vez não proporcionou secagem do referido material antes de armazená-lo.

$\mathrm{O}$ farelo (Épocas V e VI) apresentou níveis mais baixos e mais homogêneos de aflatoxina, pouco variando o teor de uma amostra para outra. Tratando-se de material mais homogêneo que o amendoim em casca, o nível mais baixo de aflatoxina nele encontrado, quer nos parecer, deva-se a uma mistura de material menos tóxico do início do processamento com material mais tóxico, mais recente ou a uma degradação térmica parcial durante o processamento.

A análise estatística revela que houve diferenças significativas, ao nível de $1 \%$ de probabilidade entre as Épocas, entre os Estágios e dentro do Estágio 1. Dentro dos outros Estágios não houve diferenças significativas. O estudo da variação da aflatoxina, em função do tempo, empregando-se regressão, revelou que houve diferença entre as Épocas, significativa ao nível de $1 \%$ de probabilidade, variação esta tendendo muito mais para curva que linear, haja visto que o valor de F (QUADRO VII), para o efeito linear, não foi significativo, ao passo que, para o efeito quadrático, foi significativo ao nível de $0,1 \%$ de probabilidade, enquadrando-se numa equação de segundo grau, do tipo da parábola. A curva de regressão pode ser observada na FIGURA 1.

O desvio da regressão não foi significativo, o que indica que a equação foi adequada.

Dos resultados obtidos neste trabalho pôde-se chegar às seguintes conclusões:

1) A maioria das amostras, representando $90 \%$ do total, continha aflatoxina.

2) O nível, em termos de aflatoxina $\mathrm{B}_{1}$, foi elevado com $60 \%$ das amostras ultra passando $1,00 \mathrm{ppm}$, considerado muito alto.

3) Os níveis de aflatoxina subiram da Época I até a Época IV: médias de 0,10 a $2,49 \mathrm{ppm}$, decrescendo nas Épocas V e VI: médias 1,02 e $1,25 \mathrm{ppm}$, com média geral de $1,23 \mathrm{ppm}$.

4) O lavrador entrega o amendoim já tóxico e muitos com elevada umidade à fábrica.

5) O armazenamento na fábrica, por esta não promover a secagem do amendoim úmido, contribui para elevação dos níveis de toxidez. 


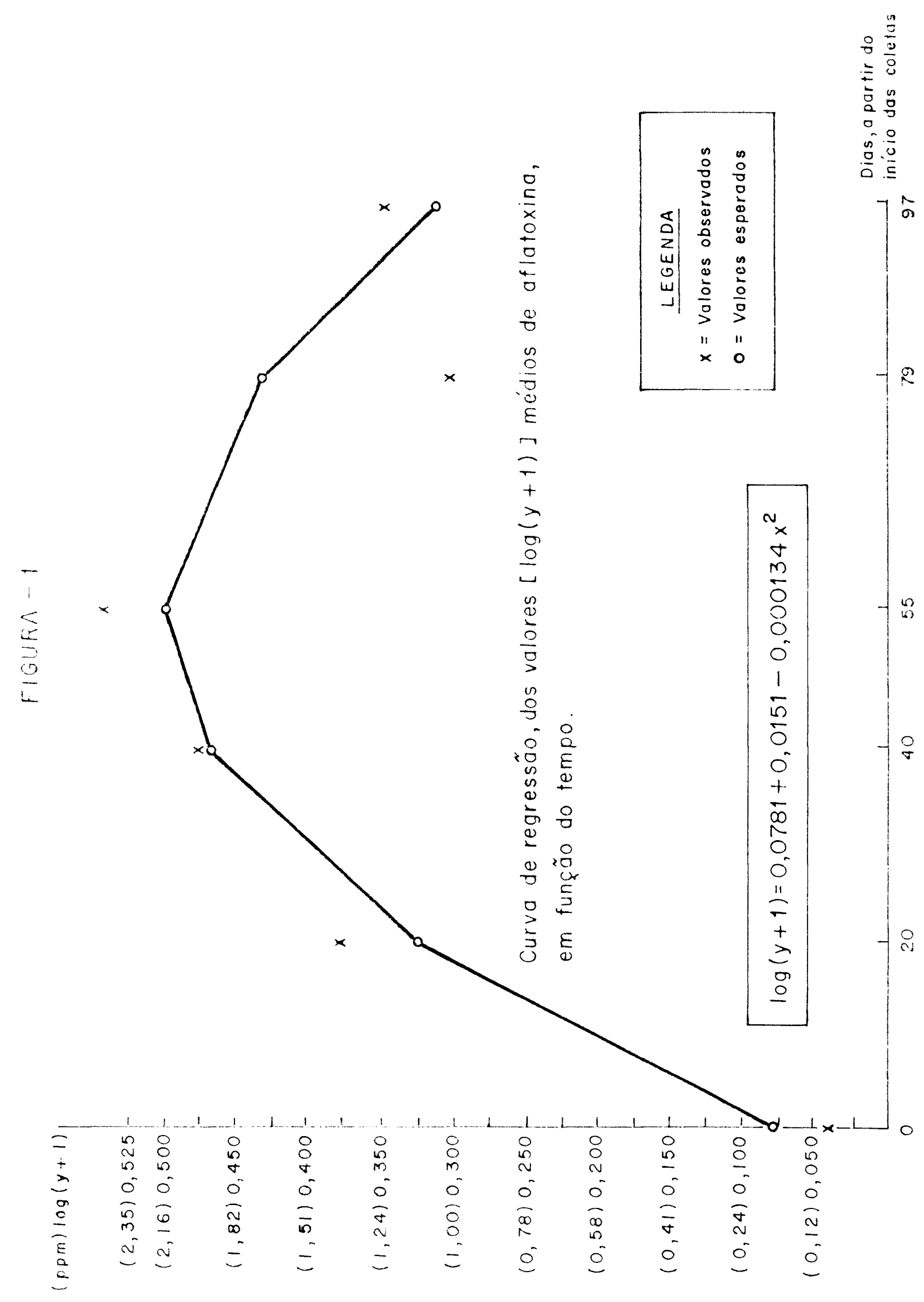




\section{SUMMARY}

\section{STUDY OF AFLATOXIN IN PEANUTS, FROM HARVEST TO INDUS- TRIALIZATION, IN THE REGION OF MONTE ALTO, S.P.}

In the present work the occurrence of aflatoxin in peanut (Arachis hypogaea L.) in the region of Monte Alto, S.P., was investigated in three Stages, from harvest to industrialization: a) by the time the grower sells it to the oil mill: Epochs I and II; b) during its storage prior to milling; Epochs III and IV and c) after oil extraction (peanut flour): Epochs V and VI. In each Stage two collections, of 10 samples each, were made in a total of 40 samples of peanuts and 20 samples of peanut flour.

From the results the following conclusions could be drawn: 1) the majory of the samples, representing $90 \%$, was toxic; 2) the toxicity level, in terms of aflatoxin $B_{1}$, was hight for $60 \%$ of the samples had more than $1.00 \mathrm{ppm}$, in the category "Very Hight"; 3) the toxicity level of the samples grew from Epoch I to Epoch IV : mean values of 0.10 to 2.49 ppm, decreasing in Epochs V and VI: 1.02 and $1.25 \mathrm{ppm}$, giving the average value of $1.23 \mathrm{ppm}$; 4) aflatoxin is already present in peanuts when the grower take it to the oil mill; 5) storage at the oil mill secms to contribute to increase the toxicy level.

\section{AGRADECIMENTOS}

Agradecemos à FAPESP pelo auxílio financeiro concedido e ao Prof. Roland Vencovsky pela orientação recebida na excursão das análises estatísticas.

\section{LITERATURA CITADA}

ASPLIN, F.D. e R.B.A. CARNAGHAN, 1961. The toxicity of certain groundnut meals for poultry with special reference to their effect on ducklings and chickens. Vet. Rec. 73 (46) : 1215-19.

BLOUNT, W.P., 1961. Turkey "X" disease. Turkeys, 9 (2) : 52, 55-58, $61,67$.

CARNAGHAN, R.B.A., R.D. HARTLEY e J. O'KELLY, 1963. Toxicity and fluorescence properties of the aflatoxins. Nature 200: 1101 .

COOMES, T.J. e A.J. FEUELL, 1965. Recommended procedures for the detection and estimation of aflatoxin $B_{1}$ in grondnuts and groundnut materials. Tropical Products Institute, Report N. ${ }^{\circ}$ G13, Ministry of Overseas Development, Londres.

DUTTON, M.F. e J.G. HEATHCOTE, 1969. Some interesting relationship between the new aflatoxins and their associated metabolites. J. South African Chem. Inst. XXII: 5107-5118.

FONSECA, H. Contribuição ao estudo da ocorrência da aflatoxina em tortas, farelos e farinhas de amendoim (Arachis hypogaea L.) do Estado de São Paulo. ESALQ/USP, Piracicaba, 65 p. Tese de Doutoramento. 
LEE, W.V., 1965. Quantitative determination of aflatoxin in groundnut products. Analyst, 90 (1070) : 305-307.

MENEZES, T.J.B.; J.S. TANGO; F.A.S. COELHO e C.G. TEIXEIRA, 1966. Ocorrência de Aspergillus Flavus e da aflatoxina em sementes e farelo de anendoim. XVIII Reunião Anual da S.B.P.C., Blumenau, S.C.

SARGEANT, K.; A. SHERIDAN; J.O'KELLY e R.B.A. CARNAGHAN, 1961. Toxicity associated with certain samples of groundnuts. Nature, 192: 1096-1097.

SNEDECOR, G.W., 1956. Statistical Methods. 5. ${ }^{\mathrm{a}}$ ed., The Iowa State College Press, Ames, Ia., $534 \mathrm{p}$.

STEEL, R.G.D. e J.H. TORRIE, 1960. Principles and Procedures of Statistics. McGraw-Hill, N. York, 481 p.

STEVENS, A.J.; C.N. SAUNDERS; J.B. SPENCE e A.C. NEWHAM, 1960. Investigations into "diseases" of turkey poults. Vet. Rec. 72 (31) : 627-628.

TANGO, J.S.; T.J.B. MENEZES e C.G. TEIXEIRA, 1967. Levantamento da ocorrência da aflatoxina em sementes de amendoim nas safras das águas e da seca de 1965. XIX Reunião Anual da S.B.P.C., Rio de Janeiro, R.J.

TROPICAL PRODUCTS INSTITUTE, 1962. Aflatoxin in groundnuts and groundnut products. Interpretation of physico-chemical and biological test results. T.P.I., Ministry of Overseas Development, Londres, 1 p. 